\title{
Literature survey, bibliographic analysis and a taxonomic catalogue of subterranean fauna from Portugal
}

\author{
Ana Sofia P. S. REBOLEIRA ${ }^{(1,2, *)}$, Fernando GONÇALVES ${ }^{(1)}$, Pedro OROMÍ (2) \\ (1) Departamento de Biologia \& Centro de Estudos do Ambiente e do Mar, Universidade de Aveiro, Campus Universitário de San- \\ tiago, 3810-193 Aveiro. Portugal, e-mail: sreboleira@ua.pt \\ (2) Departamento de Biología Animal, Facultad de Biología, Universidad de La Laguna. 38206 La Laguna. Tenerife. Islas Canarias, \\ Spain \\ * corresponding author
}

\begin{abstract}
A bibliographic analysis of the hypogean biological studies in Portugal is made, compiling 138 publications related to the subterranean invertebrate fauna, since its begining in 1870 until November 2012.

A catalogue of hypogean endemic taxa is provided, listing 27 troglobionts and 63 stygobionts, described to be obligate hypogean and endemic from mainland Portugal (Macaronesian archipelagos excluded).

The first impetus on troglobiont studies was provided by the prospections of Barros Machado during 1940's and by an expedition of Lindberg in the spring of 1961; and the major information about stygobiont species was provided by the former Instituto de Zoologia "Dr. Augusto Nobre" from Porto University.
\end{abstract}

Key words: Bibliography, checklist, troglobionts, stygobionts, Portugal

\section{INTRODUCTION}

A literature survey allows a critical assessment of the state of subterranean biology, and is a crucial labor to put order in the knowledge of the hypogean fauna, and to avoid repetition of research due to the difficulties of getting information in old bibliographical sources (Fan 2006).

The subterranean habitat harbours an extremely specialized fauna, being a true laboratory for the study of ecology, natural selection and evolution, central subjects in biology (Culver \& Pipan 2009). The study of the subterranean fauna began limited to the perspective of 'human size' caves as the domain of the cave-dwelling fauna (Vandel 1964), but the evolution of knowledge in subterranean biology leads to a broad view of the subterranean habitats as a large variety of places with aphotic conditions, extending their limits far beyond the caves (Pipan \& Culver 2012).

The first published register of subterranean fauna from Portugal (Chiroptera excluded) was in the monography "Trechorum oculatorum" where specimens of a ground beetle collected by Schaufuss in portuguese caves were recorded by Putzeys (1870: 18). The specimens were assigned to Trechus fulvus Dejean, 1831 in spite of exhibiting a larger and more rounded pronotum than is proper to this species, and neither the caves nor the geographic area of Portugal were specified.

The first hypogean taxon described from Portugal was the troglobiont spider Nesticus lusitanicus Fage, 1931, based on specimens collected during a cave survey and exploration by the archaeologist Henri Breuil and the geologist Ernest Fleury, in the beginings of the last century (Gama \& Afonso 1994).
The great momentum in the history of Portuguese subterranean biology was given by António de Barros Machado, an eminent arachnologist that began methodical exploration of caves in Portugal in the late 1930's. He performed an intense sampling effort on terrestrial hypogean fauna along the karstic caves of Portugal, and co-authored by his brother Bernardino de Barros Machado described more than 300 caves in the "Inventário das cavernas calcárias de Portugal". In the same decade, Fernando Frade from Lisbon University described Proasellus lusitanicus (Frade, 1938) from the Alviela spring in the Estremenho karst massif, paving the way for the study of stygobionts in the following decades by researchers of the former Instituto de Zoologia "Dr. Augusto Nobre" from Porto University.

Most of the biological hypogean studies in Portugal have been done in caves and dark wells. A large cryptic biodiversity still remains to unravel, especially in the mesocavernous shallow substratum (MSS as described by Juberthie et al 1980) and in the anchialine waters of coastal karsts.

More recently several efforts by Reboleira and colleagues, among others, to access subterranean biodiversity in Portugal in karst areas are providing new interesting information (Reboleira et al 2011).

\section{METHODS}

The entire bibliography on Portuguese subterranean biology has been revised (excluding that on Macarone- 
sian archipelagos and references to Chiroptera) since the first publication in 1870 until November 2012.

The term hypogean is used for the species that live exclusively in subterranean habitats beneath the soil layers, displaying clear adaptations to the subterranean environment, expressed morphologically as convergent evolution, such as integument depigmentation, absence or reduced ocular structures, elongated appendages, and wing reduction in insects. The endogean fauna occurring inside the soil is excluded of the aim of this catalogue.

\section{RESULTS}

As much as 138 scientific publications have been published between 1870 and November 2012, dealing on the hypogean fauna of mainland Portugal, from where 27 troglobiont and 63 stygobiont species have been described so far (see Table 1 and 2 for taxonomic information).
Chronologically, the proportion of higher number of publications is not necessarily the result of higher number of new described taxa (Fig. 1).

The decade of 1960 was the most productive in number of such publications, but the largest number of new hypogean taxa were described in the decades of 1940 and especially 1980 (Fig. 1).

The knowledge of the Portuguese stygofauna increased noticeably from the 40 's until the 60 's, but the greatest expansion was in the 80's with the description of 19 new species (Fig. 1). The researchers of the former Instituto de Investigação "Dr. Augusto Nobre" from Porto University gave the greatest contribution to the description of new species, being only produced out of this institute $11 \%$ of this knowledge. José Maria Braga (25 species) and Odette Afonso (21 species) contributed to 74\% of the Portuguese stygobiont species (Fig. 2).

The 1940's were the most productive decade of taxonomical studies on the terrestrial hypogean fauna as a re-

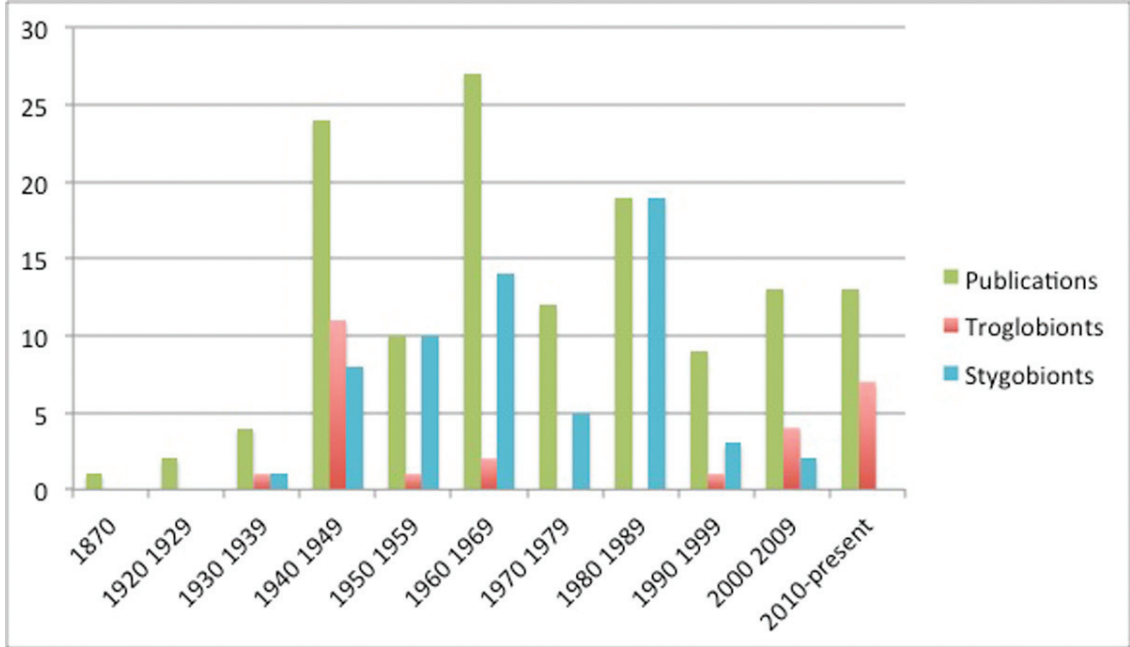

Fig. 1 - Number of publications and described hypogean taxa per decade until August 2012.

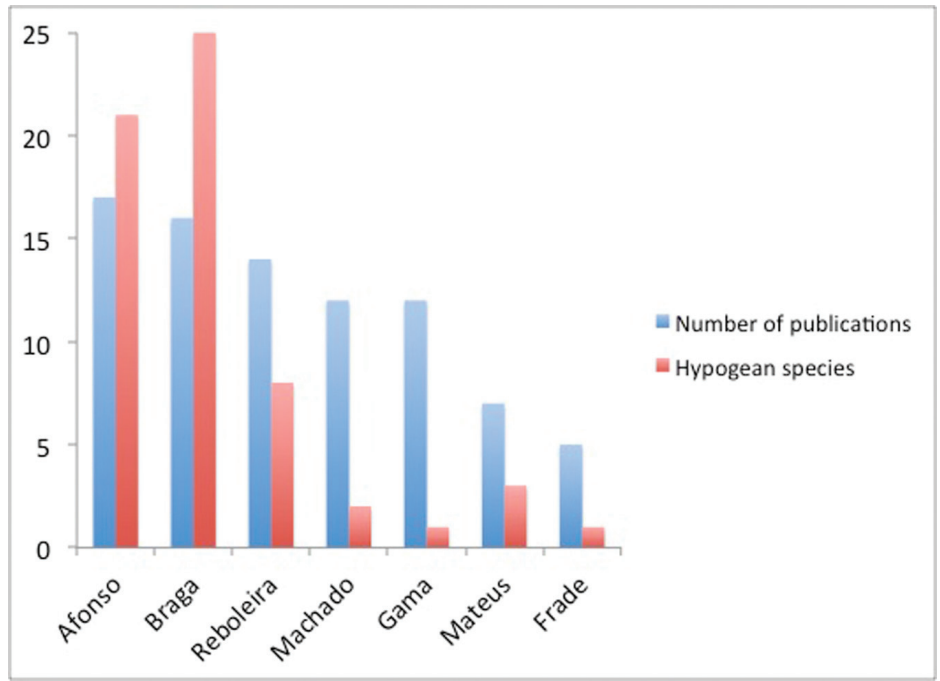

Fig. 2 - Top contributors first authors with five or more publications on hypogean fauna in Portugal and their number of described species until November 2012. 

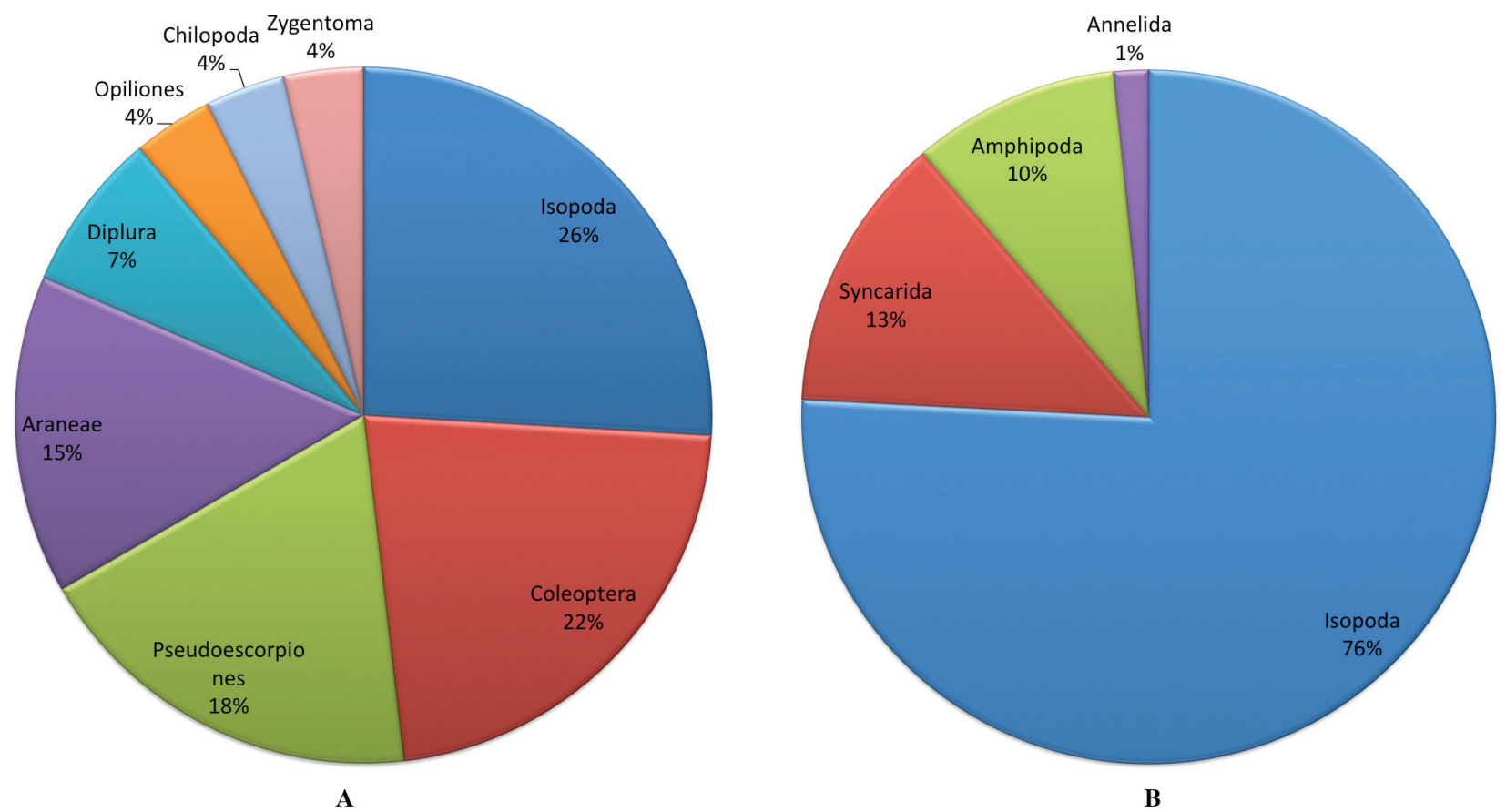

Fig. 3 - Number of hypogean species per higher taxonomic groups, until November 2012. A) Troglobionts and B) Stygobionts.

sult of the great sampling efforts of Barros Machado (Fig 2 ), but the activity almost stopped during the 70's and the 80 's. The most important contribution in the past, in number of new troglobiontic taxa was afforded by Vandel, who described 6 species of terrestrial isopods (Vandel 1946) (Fig. 3). Isopods are the most diverse group (Fig. 3 ) in the Portuguese subterranean fauna, characterized by the absence of hypogean species belonging to groups normally with troglobionts in neighbouring Spain, such as Gastropods, Palpigrads, Diplopods, Leptodirine beetles, and other Arthropod groups.

\section{ACKNOWLEDGEMENTS}

We would like to express our gratitude to F. Tatá Regala, V.M. Ortuño and Luís Mendes for kindly providing some bibliography. The Portuguese Foundation for Science and Technology (FCT, Portugal) financed Ana Sofia Reboleira by means of a Ph.D. grant (SFRH/ $\mathrm{BD} / 45744 / 2008$ ) and within the framework of KarstRisk project (PTDC/AAC-AMB/114781/2009).

\section{REFERENCES}

Culver, D.C., T. Pipan. 2009. Biology of caves and other subterranean habitats. Oxford: Oxford University Press.

Fan, Q.H. 2006. Bibliographic analysis of acarological papers published in Zootaxa from 2001 to 2005, with a catalogue of described new taxa. Zootaxa 1385: 53-66.
Juberthie, C., D. Delay, M. Bouillon. 1980. Extension du milieu souterrain en zone non-calcaire: description d'un nouveau milieu et de son peuplement par les Coléoptéres troglobies. Mémoires de Biospéologie 7: 19-52.

Pipan, T., D.C. Culver. 2012. Convergence and divergence in the subterranean realm: a reassessment. Biological Journal of the Linnean Society 107: 1-14.

Reboleira A.S.P.S., P.A.V. Borges, F. Gonçalves, A. Serrano, P. Oromí. 2011. The subterranean fauna of a biodiversity hotspot region - Portugal: an overview and its conservation. International Journal of Speleology 40, 1: 23-37.

Vandel, A. 1964. Biospéologie: La Biologie des Animaux cavernicoles. Paris: Ed. Gauthier-Villars.

\section{BIBLIOGRAPHIC CATALOGUE OF SUBTERRA- NEAN FAUNA FROM PORTUGAL}

\section{A}

Afonso, O. 1978a. Une nouvelle espèce d'Isopode troglobie du Portugal. Publicações do Instituto de Zoologia «Dr. Augusto Nobre», 61: 11-23.

Afonso, O. 1978b. Amphipoda hypogés du Portugal. Publicações do Instituto de Zoologia "Dr. Augusto Nobre", 142: 11-26.

Afonso, O. 1979. Un Proasellus (Crust. Isopoda) nouveau du Portugal. Anais da Faculdade de Ciências Universidade do Porto, 61(1-4): 91-104.

Afonso, O. 1982a. Description d'une nouvelle espèce de Proasellus (Crust. Isopoda) du Portugal et des considérations sur la phylogénie de son genre. Publicações do Instituto de Zoologia «Dr. Augusto Nobre», 163: 1-15. 
Afonso, O. 1982b. Sur un Proasellus (Crustacea, Isopoda, Asellidae) cavernicole nouveau du Portugal. Publicações do Instituto de Zoologia «Dr. Augusto Nobre», 166: 1-10.

Afonso, O. 1982c. Un nouvel asellide cavernicole du Portugal: Proasellus mateusorum sp. n. (Crustacea, Isopoda, Asellota). Publicações do Instituto de Zoologia “Dr. Augusto Nobre”, 168: 1-10.

Afonso, O. 1982d. Contribution à l'étude des Asellides (Crustacea, Isopoda) du Portugal - description d'une nouvelle espèce. Publicações do Instituto de Zoologia «Dr. Augusto Nobre», 172: 1-20.

Afonso, O. 1983. Un aselle phréatique nouveau du bassin du Mondego (Portugal) et des considerations sure les Asellides Portugais (Crust., Isopoda, Asellidae). Publicações do Instituto de Zoologia «Dr. Augusto Nobre», 178: 1-14.

Afonso, O. 1984a. Un nouveau Synasellus (Crust., Isopoda, Asellidae) de la nape phréatique littorale au sud du Douro. Publicações do Instituto de Zoologia «Dr. Augusto Nobre», 185: 1-12.

Afonso, O. 1984b. Bragasellus incurvatus sp. n. (Crustacea, Isopoda, Asellidae) nouvelle espèce hypogée du bassin du Douro. Publicações do Instituto de Zoologia «Dr. Augusto Nobre», 187: 1-9.

Afonso, O. 1985. Un asellide nouveau (Crustacea, Isopoda) de la nappe phréatique du Douro: Synasellus intermedius sp. n.. Publicações do Instituto de Zoologia «Dr. Augusto Nobre», 188: 1-8.

Afonso, O. 1987a. Un nouveau phréatobie du nord du Portugal, Synasellus bragai sp. n. (Crustacea, Isopoda, Asellidae). Algar, 1: 39-46.

Afonso, O. 1987b. Contribuition pour la connaissance des rapports entre la qualité de l'eau phréatique et les communautés d'Asellides (Crustacea, Isopoda, Asellota). Publicações do Instituto de Zoologia «Dr. Augusto Nobre», 198: 1-61.

Afonso, O. 1988. Isopoda Asellidae e Amphipoda da Gruta da Assafora (Portugal) - descrição de Proasellus assaforensis sp. n.. Algar, 2: 43-50.

Afonso, O. 1992. Aselídeos (Crustacea, Isopoda) nas águas subterrâneas portuguesas. Aspectos taxonómicos, bio-geográficos e ecológicos. Algar, 3: 49-56.

Afonso, O. 1996. Isopodes (Crustacea, Asellidae) stygobies du Nord du Portugal. Description de trois nouvelles espèces. Publicações do Instituto de Zoologia "Dr. Augusto Nobre", 235: 1-14.

Almeida, M.G., I.R. Amorim, H.M. Carvalho, N.J. Farinha, C.M. Franco, C.M. Quaresma. 1994. Contribuição para o conhecimento da flora micológica da cavidade Fojo dos morcegos (Serra da Arrábida, Portugal). Revista Biologia (Lisboa), 15: 173-179.

\section{B}

Beier, M. 1962. Eine Neue Microcreagris (Roncocreagris) aus Portugal. Voyage au Portugal du Dr. K. Lindberg. Resultats Zoologiques I. Boletim da Sociedade Portuguesa de Ciências Naturais, 9 (2ª́rie) 24: 25-26
Bellés, X. 1987. Fauna cavernícola i intersticial de la Península Ibérica i les Illes Balears. Consejo Superior de Investigaciones Cientificas, Ed. Moll, Mallorca, 207 pp.

Bivort, B.L., G. Giribet. 2004. A new genus of cyphophthalmid from the Iberian Peninsula. Invertebrate Systematics, 18: 7-52.

Bosmans, R., P. Cardoso, L.C. Crespo. 2010. A review of the linyphiid spiders of Portugal, with the description of six new species (Araneae: Linyphiidae). Zootaxa, 2473: 1-67.

Braga, J.M. 1942a. Mancasellus mariae, a new blind Isopod inhabiting subterranean waters in Portugal. Memórias e Estudos do Museu Zoológico da Universidade de Coimbra, 130: 1-11.

Braga, J.M. 1942b. Un Isopode nouveau du Portugal: Stenasellus nobrei n. sp. Publicações do Instituto de Zoologia «Augusto Nobre», 10: 1-11.

Braga, J.M. 1943. Description de l'Asellus seabrai, isopode aveugle nouveau des eaux souterraines du Portugal. Publicações do Instituto de Zoologia «Dr. Augusto Nobre», 14: 1-17.

Braga, J.M. 1944. Note sur un genre d'Asellides nouveau du Portugal (Synasellus n. gen.) et description d'une sous-espèce nouvelle de ce genre. Publicações do Instituto de Zoologia «Dr. Augusto Nobre», 17: 1-11.

Braga, J.M. 1945. Un nouvel Aselle cavernicole du Portugal. Publicações do Instituto de Zoologia «Dr. Augusto Nobre», 24: 1-15.

Braga, J.M. 1946. Quelques Asellides nouveaux du Portugal. Synasellus fragilis n. sp., Asellus coxalis ibericus n. subsp., Asellus conimbricensis n. sp. Publicações do Instituto de Zoologia «Dr. Augusto Nobre», 29: 1-15.

Braga J.M. 1949. Un Bathynellidae (Crust. Syncarida) du Portugal (Parabathynella lusitanica n. sp.). Publicações do Instituto de Zoologia “Dr. Augusto Nobre”, 40: 1-15.

Braga, J.M. 1956. Un Asellus nouveau de l'Étang de Fermentelos (Portugal). Publicações do Instituto de Zoologia «Dr. Augusto Nobre», 53: 1-12.

Braga, J.M. 1958. Un Asellus remarquable des eaux souterraines du Portugal (Asellus pauloae n. sp.). Publicações do Instituto de Zoologia «Dr. Augusto Nobre», 61: 1-15.

Braga, J. M. 1959. Le genre Synasellus et ses espèces. Publicações do Instituto de Zoologia «Dr. Augusto Nobre», 71:1-72.

Braga, J.M. 1960a. Synasellus albicastrensis un nouveau asellide troglobie du Portugal. Anais da Faculdade de Ciências da Universidade do Porto, 43: 1-15.

Braga J.M. 1960b. Sur une Parabathynella (Crust. Syncarida) nouvelle du Portugal. Publicações do Instituto de Zoologia "Dr. Augusto Nobre”, 75: 9-22.

Braga, J.M. 1962. Sur la distribution géographique des Stenasellus de la Péninsule Ibérique et description d'une espèce nouvelle de ce genre. Anais da Faculdade de Ciencias Universidade do Porto, 84: 9-28.

Braga, J.M. 1964. Contribution à la faune d'asellides du Portugal Asellus frontellum sp. n.. Publicações do Instituto de Zoologia «Dr. Augusto Nobre», 91: 9-18. 
Braga, J.M. 1967. Trois Synasellus (Crust, Isopoda) nouveaux du Portugal: Synasellus serranus sp. n., Sunasellus nobrei sp. n., Synasellus minutus sp. n. Anais da Faculdade de Ciências da Universidade do Porto, 50(34): 301-324.

Braga, J.M. 1968. Sur un Asellide psammique nouveau du Portugal. Publicações do Instituto de Zoologia «Dr. Augusto Nobre», 102: 1-21.

Brotas, G. 2007. Comunidades de artrópodes terrestres em área cársica: estudos em meio epígeo e hipógeo. M.Sc. thesis. Universidade do Minho, Portugal.

\section{C}

Camacho, A.I. 2003a. Four new species of groundwater crustaceans (Syncarida, Bathynellacea, Parabathynellidae) endemic to the Iberian Peninsula. Journal of Natural History, 37: 2885-2907.

Camacho, A.I. 2003b. Historical biogeography of Hexabathynella, a cosmopolitan genus of groundwater Syncarida (Crustacea, Bathynellacea, Parabathynellidae). Biological Journal of the Linnean Society, 78: 457-466.

Cardoso, P., M.N. Duy-Jacquemin, F. Rasteiro. 2008. New and first records of Polyxenida (Diplopoda: Penicillata) from Portugal. Boletín Sociedad Entomológica Aragonesa, 42: 360.

Cardoso, P., N. Scharff. 2009. First record of the spider family Symphytognathidae in Europe and description of Anapistula ataecina sp. n. (Araneae). Zootaxa 2246: 45-57.

Cardoso, P. (2012) Diversity and community assembly patterns of epigean vs. troglobiont spiders in the Iberian Peninsula. International Journal of Speleology, 41(1): 83-94.

Coiffat, H. 1962. Voyage au Portugal du Dr. K. Lindberg. Résultats zoologiques. 4. Coléoptères cavernicoles. Boletim da Sociedade Portuguesa de Ciências Naturais, 9(2): 90-99.

Condé, B. 1951. Campodéidés du Portugal récoltés par M. A. de Barros Machado. Memórias e Estudos do Museu Zoológico da Universidade de Coimbra, 204, 1-12.

Condé, B. 1955. Matériaux pour une Monographie des Diploures Campodéidés. Mémoires du Muséum National d'Histoire naturelle, série A. Zoologie, 12, 1-202.

Cons, D. 1959. The caves of the Serra de Aire Massif, Central Portugal. Transactions of the Cave Research Group of Great Britain, 5(2): 61-105.

Cruz, A. 1991. Especies nuevas o poco conocidas de isópodos terrestres de la Península Ibérica. II. Isópodos epigeos de España y Portugal (Crustacea, Oniscidea). Bulletin de la Société d'Histoire naturelle de Toulouse, 127: 71-75.

\section{D}

Deboutteville, C. D. 1944. Collemboles cavernicoles du Portugal, récoltés par B. Machado (I Série). Revue Française de Entomologie, 11: 29-35.

Deboutteville, C. D. 1946. Collemboles cavernicoles du Portugal, récoltés par B. Machado (II Série). Revue Française de Entomologie, 13: 100-104.

\section{$\mathbf{E}$}

Eiras, J. 1974. Un nouveau Synasellus (Crustacea, Isopoda) du Portugal. Publicações do Instituto de Zoologia «Augusto Nobre», 124: 9-21.

\section{F}

Fage, L. 1931. Araneae, 5émé Sér. précédée d'un essai sur l'évolution souterraine et son déterminisme. Biospeologica, 55, Paris. Archives de Zoologie Experimentale et Générale, Paris, 71: 99-291.

Fernandes, J.A. 1962. A fauna subterrânea de Portugal. Boletim da Sociedade Portuguesa de Ciências Naturais, 9(24): 3-4.

Frade, F. 1938. Un Asellus (Isopode) nouveau des eaux souterraines du Portugal. Bulletin de la Société Portugaise des Sciences Naturelles, 7(5): 17-24.

Frade, F. 1939. Crustáceos das Águas do Canal do Alviela. Notas acerca do aparecimento do Asellus lusitanicus Frade. Boletim da Comissão Fiscal das Obras de Abastecimento de água à cidade de Lisboa, $\mathrm{n}^{\mathrm{o}}$ 15, Lisboa.

Frade, F. 1939. Um crustáceo cavernícola de Portugal. (Notas biológicas). Naturália, III, Lisboa.

Frade, F. 1940. Da fauna e flora das águas de abastecimento à Capital. Necessidade do seu estudo. Lisboa, 15: 267-292.

Frade, F. 1941. Os estudos hidrobiológicos e as águas de abastecimento. Boletim da Comissão Fiscal das Obras de Abastecimento de água à cidade de Lisboa, $\mathrm{n}^{\mathrm{o}} 18$.

Frade, F. 1945. Crustáceos dulciaquícolas de Portugal, II. Aselídeos das águas superficiais e subterrâneas. Naturália, 26, Lisboa.

\section{G}

Galhano, M. H. 1970. Contribução para o conhecimento da fauna intersticial em Portugal. Publicações do Instituto de Zoologia “Dr. Augusto Nobre”, 110: 9-206.

Gama, M.M. 1959. Contribuição para o estudo dos Colêmbolos de Portugal Continental. Memórias e estudos do Museu Zoológico da Universidade de Coimbra, 260.

Gama, M.M. 1962. Collemboles des grottes du Portugal. Voyage au Portugal du Dr. K. Lindberg. Résultats zoologiques 5. Boletim da Sociedade Portuguesa de Ciências Naturais, 2a serie, 9: 100-108.

Gama, M.M. 1963. Monografia do género Isotomodes (Insecta; Collembola). Memórias e estudos do Museu Zoológico da Universidade de Coimbra, nº 284.

Gama, M.M. 1964. Colêmbolos de Portugal Continental. Dissertação de Doutoramento em Ciências Biológicas. Universidade de Coimbra. 253 pp.

Gama, M.M. 1965. Colêmbolos de Portugal Continental. Memórias e estudos do Museu Zoológico da Universidade de Coimbra, 292 pp.

Gama, M.M. 1966. Notes taxonomiques sur quelques espèces de Collemboles. Memórias e Estudos do Museu Zoológico da Universidade de Coimbra, 295: 5-21.

Gama, M.M. 1969. Notes taxonomiques et lignées généalogiques de quarante deux espèces et sous-espèces 
du genre Xenylla (Insecta, Collembola). Memórias e estudos do Museu Zoológico da Universidade de Coimbra, 308: 19-27.

Gama, M.M. 1974. Systématique évolutive de quelques espèces de Pseudosinella (Collembola, Insecta) appartenant à trois lignées généalogiques. Pedobiolobia, 14: 279-284.

Gama, M.M. 1977. Systématique évolutive des Pseudosinella. XI. Espèces provenant de da Péninsule Ibérique et d'Europe Centrale (Insecta: Collembola). Revue suisse de Zoologie, 84 (3): 593-597.

Gama, M.M. 1992. A Bioespeleologia em Portugal. Actas do $3^{\circ}$ Congresso Nacional de Espeleologia.

Gama, M.M. \& Afonso, O. 1994. Portugal. In: Juberthie, C. and Decu, V. (eds.), Encyclopaedia Biospeologica, tome I. Sociétè de Biospéologie, Moulis, France, pp.771-778.

Giani, N., B. Sambugar, P. Rodríguez, E. Martínez-Ansemil. 2001. Oligochaetes in southern European groundwater: new records and an overview. Hydrobiologia, 463: 65-74.

Gisin, H. 1960. Collembolenfauna Europas. Muséum d'Histoire Naturelle, Genève, 312 pp.

Gisin, H., M.M. Gama. 1969. Espèces nouvelles de Pseudosinella cavernicoles (Insecta: Collembola). Revue Suisse de Zoologie, 76 (1): 143-181.

\section{$\mathbf{H}$}

Henry, J.P., G. Magniez. 1968. Sur la systématique et la biogeographie des Asellides. Comptes Rendues de l'Académie des Sciences, Paris, 268: 87-89.

Henry, J.P., G. Magniez. 1970. Contribution à la systématique des Asellides (Crustacea Isopoda). Annales de Spéléologie, 25(2) : 335-367.

Hrabe, S. 1963. On Rhyacodrilus lindbergi n. sp., a new cavernicolous species of the family Tubificidae (Oligochaeta) from Portugal. Boletim da Sociedade Portuguesa de Ciências Naturais, 10: 52-56.

\section{$\mathbf{J}$}

Jeannel, R. 1920. Étude sur le Trechus fulvus Dej. (Col. Carab.). Sa phylogénie, son intérêt biogéographique. Trabajos del Museo Nacional de Ciencias Naturales. Serie Zoológica, 41: 5-24.

Jeannel, R. 1941. Premières explorations des grottes du Portugal par M.A. de B. Machado, Coléoptères. Publicações do Instituto de Zoologia «Augusto Nobre», 4: 5-15.

Jeannel, R., E.G. Racovitza. 1929. Enumération des Grottes Visitées 1918-1927 (7éme Série). Biospeleologica 54. Archives de Zoologie Experimentale et Génerale, Paris, 68 : 293-608.

\section{L}

Lescher-Moutoué, F. 1981. Cyclopidae des eaux souterraines du Portugal et de l'île de Majorque (Crustacea, Copepoda). Bulletin of the Zoological Museum of Amsterdam, 8, 8: 65-67.
Lindberg, K. 1962. Cyclopides (Crustacés, Copépodes) du Portugal. Voyage au Portugal du Dr. K. Lindberg. Résultats Zoologiques 2. Boletim da Sociedade Portuguesa de Ciências Naturais, 9: 23-48.

Lindberg, K. 1962. Notes sur quelques grottes et aperçu de leur faune. Voyage au Portugal du Dr. Knut Lindberg. Résultats Zoologiques 3. Boletim da Sociedade Portuguesa de Ciências Naturais, 9: 49-89.

Lindberg, K., O. Kraus. 1963. Opilions des Grottes Portugaises. Voyage au Portugal du Dr. Knut Lindberg. Résultats Zoologiques 8. Boletim da Sociedade Portuguesa de Ciências Naturais, 10: 7-9.

Lopez-Pancorbo A., C. Ribera. 2011. Nesticus baeticus sp. n., a new troglobitic spider species from southwest Europe (Araneae, Nesticidae). ZooKeys, 89: 1-3.

\section{M}

Machado, A.B. 1941a. As cavernas de Portugal sob o ponto de vista biológico. Boletim da Sociedade Portuguesa de Ciências Naturais, 13: 639-642.

Machado, A.B. 1941b. Araignées nouvelles pour la faune portugaise. Publicações do Instituto de Zoologia «Dr. Augusto Nobre», 3: 1-60.

Machado, A.B. 1945a. Observations inédites sur le colulus et les filères de quelques Aranéides, accompagnées de notes critiques sur la morphologie comprarée des filières. Publicações do Instituto de Zoologia do Porto "Dr. Augusto Nobre”, 22: 3-44.

Machado, A.B. 1945b. À propos de l'appareil respiratoire des "Leptonetidae" (Araneae). Publicações do Instituto de Zoologia do Porto “Dr. Augusto Nobre”, 23: 131-135.

Machado, A.B. 1945c. Instruções para a Exploração Biológica das Cavernas. Publicações do Instituto de Zoologia do Porto "Dr. Augusto Nobre”, 21: 1-15.

Machado, A. B. 1946. Contribuição para o conhecimento dos miriápodes de Portugal. Publicações do Instituto de Zoologia “Dr. Augusto Nobre”, 26, 5-37.

Machado, A. B. 1948. As cavernas de Portugal sob o ponto de vista biológico. Publicações do Instituto de Zoologia "Dr. Augusto Nobre”, 37, 1-16.

Machado, A.B. 1952. Miriápodes de Portugal. Primeira parte: Quilópodes. Publicações do Instituto de Zoologia "Dr. Augusto Nobre”, 43: 1-159

Machado, A. B. 1951. Ochyroceratidae (Araneae) de l'Angola. Publicações Culturais, Companhia de Diamantes de Angola (Diamang), 8, 1-88.

Machado, A.B., B.B. Machado. 1941. Inventário das cavernas calcárias de Portugal. Boletim da Sociedade Portuguesa de Ciências Naturais, 13: 444-473.

Machado, A. B., C. Ribera. 1986. Araneidos cavernícolas de Portugal: familia Leptonetidae (Araneae). Actas X Congresso Internacional de Aracnología, 355-366.

Madeira, F. 1994. Bioespeleologia de Portugal I (Ensaio Bibliográfico) Edição de autor, 29 p. Lisboa.

Magniez, G. 1967. Geographic distribution and validity of the troglobe species Asellus lusitanicus Frade 
(Asellote Crustacean). International Journal of Speleology, 2(4): 315-317.

Mateus, A. 1957. Anfípodes Troglóbios. Publicações do Instituto de Zoologia "Dr. Augusto Nobre”, 58: 1-8.

Mateus, A. 1974. Sur la Phylogénie du genre Hadzia (Crustacea, Amphipoda). Publicações do Instituto de Zoologia "Dr. Augusto Nobre”, 128: 9-33.

Mateus, A. 1980. La faune portugaise d'Amphipodes hypogés et sa comparaison à celle de la Roumanie. Travaux de l'Institute de Spéologie «Emil Racovitza», 19.

Mateus, A., M.L. Maciel. 1967. Description d'une espéce de Bogidiella (Crustacea, Amphipoda) du psammon du Portugal et quelques notes sur son genre. Publicações do Instituto de Zoologia "Dr. Augusto Nobre”, 100: 11-47.

Mateus A., E. Mateus. 1958. Un noveau genre et une nouvelle espèce d'Amphipode troglobie du Portugal. Memórias e estudos do Museu Zoológico da Universidade de Coimbra, 252: 1-10.

Mateus A., E. Mateus. 1972. Une nouvelle espèce d'Hadzia (Crustacea: Amphipoda) du Portugal. Publicações do Instituto de Zoologia “Dr. Augusto Nobre”, 117: 9-30.

Mateus A., E. Mateus. 1978. Amphipoda hypogés du Portugal. Publicações do Instituto de Zoologia "Dr. Augusto Nobre", 142: 11-26.

Mendes, L.M. 1996. Further data on the Nicoletiidae (Zygentoma) with the description of a new species from Mauritius. Revue Suisse de Zoologie, 103(3): 749-756.

\section{N}

Noodt W., M.H. Galhano. 1969. Studien an Crustacea Subterranea (Isopoda, Syncarida, Copepoda) aus dem Norden Portugal. Publicações Instituto Zoologia "Dr. Augusto Nobre”, 107: 9-75.

Notenboom, J. 1987. Lusitanian species of the amphipod. Pseudoniphargus Chevreux, 1901 with a key to all Iberian species. Bijdr. Dierk. 57 (2): 191-206.

Notenboom, J. 1990. Introduction to Iberian groundwater amphipods. Limnetica, 6: 165-176.

$\mathbf{P}$

Putzeys, J. 1870. Trechorum oculatorum Monographia. Stettin entomologische Zeitschrift, 31: 7-48.

\section{R}

Reboleira, A.S.P.S. 2005. Qualidade da água da nascente de Anços (Serra de Sicó) com o uso de fitoperifiton. Espeleodivulgação, 6: 30-35.

Reboleira, A.S.P.S. 2007. Os Coleópteros (Insecta, Coleoptera) cavernícolas do Maciço Calcário Estremenho: uma abordagem à sua biodiversidade. M.Sc. thesis. Universidade de Aveiro, Portugal, 84 pp.

Reboleira, A.S., F.J. Gonçalves, A.R.M. Serrano. 2008. Cave Coleoptera from Estremenho Karstic Massif, Portugal. Spelunca Memories, 33: 65.

Reboleira, A.S.P.S, F. Gonçalves, A.R.M. Serrano. 2009. Two new species of cave dwelling Trechus Clair- ville, 1806 of the fulvus-group (Coleoptera, Carabidae, Trechinae) from Portugal. Deutsche Entomologische Zeitschrift, 56(1): 101-107.

Reboleira A.S.P.S., F. Gonçalves, P. Oromí, J.A. Zaragoza 2010a. Titanobochica, surprising discovery of a new cave-dwelling genus from southern Portugal (Arachnida: Pseudoscorpiones: Bochicidae). Zootaxa, 2681: 1-19.

Reboleira A.S.P.S., V.M. Ortuño, F. Gonçalves, P. Oromí. 2010b. A hypogean new species of Trechus Clairville, 1806 (Coleoptera, Carabidae) from Portugal and considerations about the T. fulvus species group. Zoota$x a, 2689$ : 15-26.

Reboleira A.S.P.S., A. Sendra, F. Gonçalves, P. Oromí. 2010c. The first hypogean dipluran from Portugal: description of a new species of the genus Litocampa (Diplura: Campodeidae). Zootaxa, 2728: 50-56.

Reboleira, A.S.P.S., V.M. Ortuño. 2011a. Description of the larva and female genitalia of Trechus gamae with data on its ecology. Bulletin of Insectology, 64(1): 43-52.

Reboleira A.S.P.S., Borges, P.A.V., Gonçalves, F., Serrano, A., Oromí, P. 2011b. The subterranean fauna of a biodiversity hotspot region - Portugal: an overview and its conservation. International Journal of Speleology, 40,(1): 23-37.

Reboleira A.S.P.S., F. Gonçalves. 2011c. Livro de resumos do II Encontro Ibérico de Biologia Subterrânea. Universidade de Aveiro. 54 pp.

Reboleira A.S.P.S., F. Gonçalves, P. Oromí. 2011d. On the Iberian endemic subgenus Lathromene Koch (Coleoptera: Staphylinidae: Paederinae): description of the first hypogean Domene Fauvel, 1872 from Portugal. Zootaxa, 2780: 48-56.

Reboleira, A.S.P.S. 2012. Biodiversity and conservation of subterranean fauna of Portuguese karst. Ph.D. Thesis. University of Aveiro, Portugal, 333 pp.

Reboleira, A.S.P.S., F. Gonçalves, P. Oromí, L.M. Mendes. 2012a. Squamatinia algharbica gen. n. sp. n., a remarkable new Coletiniinae silverfish (Zygentoma: Nicoletiidae) from caves in southern Portugal Zootaxa, 3260: 33-46.

Reboleira A.S.P.S., J.A. Zaragoza, F. Gonçalves, P. Oromí. 2012b. Lusoblothrus, a new syarinid pseudoscorpion genus (Arachnida) from Portugal, occupying an isolated position within the Holarctic fauna. Zootaxa, 3544: 52-62.

Ribera, C. 1988a. La familia Leptonetidae (Arachnida, Araneae) in la Península Ibérica. In Haupt, J. (ed.), XI Europäisches Arachnologisches Colloquium. Technische Universität Berlin Dokumentation Kongresse und Tagungun, 38: 267-281.

Ribera, C. 1988b. Descripción del macho de Nesticus lusitanicus Fage 1931. (Araneae, Nesticidae). Publicaciones del Departamento de. Zoología de la Universidad de Barcelona, 14: 37-42.

Ribera, C. 1993. Dysdera caeca n.sp. y Harpactea stalitoides n.sp. (Araneae), dos especies cavernícolas de Marruecos y Portugal. Revue Arachnologique, 10(1): 1-7. 
Sendra A., A. Achurra, P. Barranco, P. Beruete, P.A.V. Borges, J.J. Herrero-Borgoñón, A.I. Camacho, C. Galán, L. García, D. Jaume, R. Jordana, J. Modesto, M.A. Monsalve, P. Oromí, V.M. Ortuño, C. Prieto, A.S. Reboleira, P. Rodríguez, J.M. Salgado, S. Teruel, A. Tinaut, J.A. Zaragoza. 2011. Biodiversidad, regiones biogeográficas y conservación de la fauna subterránea Hispano-lusa. Boletín de la Sociedad Aragonesa de Entomología, 49: 365-400.

Stock, J.H. 1980. Regression model evolution as exemplified by the genus Pseudoniphargus (Amphipoda). Bijdragen tot de Dierkunde, 50 (1): 105-144.

\section{T}

Thunberg, E. 1963. Diptères Dolichopodidade. Voyage au Portugal du Dr. K. Lindberg. Résultats Zoologiques 7. Boletim da Sociedade Portuguesa de Ciências Naturais, 10(25): 5-6.

Tollet, R. 1948. Explorations Biologiques des Grottes du Portugal. Publicações do Instituto de Zoologia "Dr. Augusto Nobre”, 35: 7-10.

\section{V}

Vachon, M. 1940. Éléments de la faune Portugaise des Pseudoscorpions (arachnides) avec description de quatre espèces nouvelles. Publicações do Instituto de Zoologia «Augusto Nobre», 2: 7-30.

Vachon, M. 1946. Description d'une nouvelle espèce de Pseudoscorpion (arachnide) habitant les grottes Portugaises: Microcreagris cavernicola. Bulletin du Muséum National d'Histoire Naturelle, $2^{\text {a }}$ Série, 18: 333-336.

Vailant, F. 1963. Diptères Psychodidae et Empididae. Voyage au Portugal du Dr. K. Lindberg. Résultats Zoologiques 6. Boletim da Sociedade Portuguesa de Ciências Naturais, 10(25): 1-4.

Vandel, A. 1946. Crustacés Isopodes terrestres (Oniscoidea) epigés et cavernicoles du Portugal. Publicações do Instituto de Zoologia "Dr. Augusto Nobre", 30: 137-437.

Vives, E. 1975. Coleópteros cavernícolas nuevos o interesantes de la Península Ibérica y Baleares. Speleon, 22: $159-169$.

\section{Z}

Zaragoza, J.A. 2007. Catálogo de los Pseudoescorpiones de la Península Ibérica e Islas Baleares (Arachnida: Pseudoscorpiones). Revista Ibérica de Aracnología, 13: 3-91.

Zaragoza, J.A. 2012. Chthonius (Ephippiochthonius) cardosoi, a new hypogean species from Portugal (Pseudoescorpiones: Chthoniidae). Revista Ibérica de Aracnología, 20: 25-30.

\section{CATALOGUE OF THE DESCRIBED HYPOGEAN ENDEMIC TAXA FROM PORTUGAL}

Table 1 - Troglobionts from Portugal (Macaronesian archipelagos excluded) described until November 2012.

\begin{tabular}{|c|c|c|}
\hline Order & Family & Species \\
\hline \multirow[t]{6}{*}{ Pseudoscorpiones } & Chthoniidae & Chthonius minutus Vachon, 1940 \\
\hline & & Chthonius cardosoi Zaragoza, 2012 \\
\hline & Bochicidae & Titanobochica magna Zaragoza \& Reboleira, 2010 \\
\hline & Neobisiidae & Roncocreagris blothroides (Beier, 1962) \\
\hline & & Roncocreagris cavernicola (Vachon, 1946) \\
\hline & Syarinidae & Lusoblothrus aenigmaticus Zaragoza \& Reboleira, 2012 \\
\hline \multirow[t]{4}{*}{ Araneae } & Dysderidae & Harpactea stalitoides Ribera, 1993 \\
\hline & Leptonetidae & Teloleptoneta synthetica (Machado, 1951) \\
\hline & Nesticidae & Nesticus lusitanicus Fage, 1931 \\
\hline & Symphytognathidae & Anapistula ataecina Cardoso \& Scharff, 2009 \\
\hline Opiliones & Sironidae & Iberosiro distylos Bivort \& Giribet, 2004 \\
\hline Lithobiomorpha & Lithobiidae & Lithobius dimorphus Machado, 1946 \\
\hline \multirow[t]{7}{*}{ Isopoda } & Porcellionidae & Porcellio cavernicolous Vandel, 1946 \\
\hline & Trichoniscidae & Trichoniscoides broteroi Vandel, 1946 \\
\hline & & Trichoniscoides subterraneus Vandel, 1946 \\
\hline & & Trichoniscoides meridionalis (Vandel, 1946) \\
\hline & & Trichoniscoides ouremensis (Vandel, 1946) \\
\hline & & Trichoniscoides serrai Cruz, 1993 \\
\hline & Armadillidiidae & Troglarmadillidium machadoi Vandel, 1946 \\
\hline Entomobryomorpha & Onychiuridae & Onychiurus confugiens Gama, 1962 \\
\hline Diplura & Campodeidae & Litocampa mendesi Sendra \& Reboleira, 2010 \\
\hline Zygentoma & Nicoletiidae & Squamatinia algharbica Mendes \& Reboleira, 2012 \\
\hline Coleoptera & Carabidae & Trechus machadoi Jeannel, 1942 \\
\hline
\end{tabular}




\begin{tabular}{|l|l|l|}
\hline Order & Family & Species \\
\hline & & Trechus gamae Reboleira \& Serrano, 2009 \\
\hline & & Trechus lunai Reboleira \& Serrano, 2009 \\
\hline & & Trechus tatai Reboleira \& Ortuño, 2010 \\
\hline & Staphylinidae & Domene lusitanica Reboleira \& Oromí, 2011 \\
\hline
\end{tabular}

Table 2 - Stygobionts from Portugal (Macaronesian archipelagos excluded) described until November 2012.

\begin{tabular}{|c|c|c|}
\hline Order & Family & Species \\
\hline Tubificida & Tubificidae & Rhyacodrilus lindbergi Hrabe, 1963 \\
\hline \multirow[t]{8}{*}{ Syncarida } & Parabathynellidae & Hexabathynella minuta (Noodt \& Galhano, 1969) \\
\hline & & Iberobathynella lusitanica (Braga, 1949) \\
\hline & & Iberobathynella barcelensis (Noodt \& Galhano, 1969) \\
\hline & & Iberobathynella valbonensis (Galhano, 1970) \\
\hline & & Iberobathynella gracilipes (Braga, 1960) \\
\hline & & Iberobathynella cavadoensis (Noodt \& Galhano, 1969) \\
\hline & & Iberobathynella serbani Camacho, 2003 \\
\hline & & Iberobathynella pedroi Camacho, 2003 \\
\hline \multirow[t]{6}{*}{ Amphipoda } & Melitidae & Pseudoniphargus mateusorum Stock, 1980 \\
\hline & & Pseudoniphargus longispinum Stock, 1980 \\
\hline & & Pseudoniphargus callaicus Notenboom, 1987 \\
\hline & Niphargidae & Haploginglymus bragai Mateus \& Mateus 1958 \\
\hline & & Metahadzia tavaresi (Mateus \& Mateus 1972) \\
\hline & Bogidiellidae & Bogidiella helenae Mateus \& Maciel, 1967 \\
\hline \multirow[t]{28}{*}{ Isopoda } & Asellidae & Bragasellus seabrai (Braga, 1943) \\
\hline & & Bragasellus conimbricensis (Braga, 1946) \\
\hline & & Bragasellus frontellum (Braga, 1964) \\
\hline & & Bragasellus incurvatus Afonso, 1984 \\
\hline & & Bragasellus pauloae (Braga, 1958) \\
\hline & & Proasellus arthrodilus (Braga, 1945) \\
\hline & & Proasellus assaforensis Afonso, 1988 \\
\hline & & Proasellus exiguus Afonso, 1983 \\
\hline & & Proasellus lusitanicus (Frade, 1938) \\
\hline & & Proasellus mateusorum Afonso, 1982 \\
\hline & & Proasellus nobrei Braga, 1942 \\
\hline & & Proasellus rectangulatus Afonso, 1982 \\
\hline & & Proasellus rectus Afonso, 1982 \\
\hline & & Proasellus spinipes Afonso, 1979 \\
\hline & & Proasellus variegatus Afonso, 1982 \\
\hline & & Psammasellus capitatus Braga, 1968 \\
\hline & & Synasellus albicastrensis Braga, 1960 \\
\hline & & Synasellus barcelensis Noodt \& Galhano, 1969 \\
\hline & & Synasellus bragai Afonso, 1987 \\
\hline & & Synasellus brigantinus Braga, 1959 \\
\hline & & Synasellus capitatus (Braga, 1968) \\
\hline & & Synasellus dissimilis Afonso, 1987 \\
\hline & & Synasellus exiguus Braga, 1944 \\
\hline & & Synasellus flaviensis Afonso, 1996 \\
\hline & & Synasellus fragilis (Braga, 1946) \\
\hline & & Synasellus henrii Afonso, 1987 \\
\hline & & Synasellus insignis Afonso, 1984 \\
\hline & & Synasellus intermedius Afonso, 1985 \\
\hline
\end{tabular}




\begin{tabular}{|c|c|c|}
\hline Order & Family & Species \\
\hline & & Synasellus lafonensis Braga, 1959 \\
\hline & & Synasellus longicauda Braga, 1959 \\
\hline & & Synasellus longicornis Afonso, 1978 \\
\hline & & Synasellus mariae (Braga, 1942) \\
\hline & & Synasellus mateusi Braga, 1954 \\
\hline & & Synasellus meirelesi Braga, 1959 \\
\hline & & Synasellus minutus Braga, 1967 \\
\hline & & Synasellus nobrei Braga, 1967 \\
\hline & & Synasellus pireslimai Braga, 1959 \\
\hline & & Synasellus robusticornis Afonso, 1987 \\
\hline & & Synasellus serranus Braga, 1967 \\
\hline & & Synasellus tirsensis Afonso, 1987 \\
\hline & & Synasellus transmontanus Braga, 1959 \\
\hline & & Synasellus valpacensis Afonso, 1996 \\
\hline & & Synasellus vidaguensis Afonso, 1996 \\
\hline & & Synasellus vilacondensis Afonso, 1987 \\
\hline & & Synasellus pombalensis Afonso, 1987 \\
\hline & & Synasellus favaiensis Eiras, 1974 \\
\hline & Stenasellidae & Stenasellus galhanoae Braga, 1962 \\
\hline & & Stenasellus nobrei Braga, 1942 \\
\hline
\end{tabular}

